\title{
Relação entre massa corporal e taxa respiratória em Arthropoda de Floresta Atlântica (Paraíba, Brasil)
}

\author{
Idalio Amaranto ${ }^{1,2}$, Cleber Ibraim Salimon ${ }^{3,4} \&$ Douglas Zeppelini ${ }^{1,2}$
}

(1) Universidade Federal da Paraíba - Campus I, Centro de Ciências Exatas e da Natureza, Departamento de Sistemática e Ecologia, Programa de Pós-Graduação em Ciências Biológicas (Zoologia), Cidade Universitária 58051-900, João Pessoa, Paraíba, Brasil. E-mail: idalio.amaranto@gmail.com

(2) Universidade Estadual da Paraíba, Centro de Ciências Biológicas e Sociais Aplicadas, Departamento de Biologia, Laboratório de Sistemática de Collembola e Conservação, Rua Horácio Trajano de Oliveira, Cristo Redentor 58071-160, João Pessoa, Paraíba, Brasil. E-mail: zeppelini1969@yahoo.com.br

(3) Universidade Estadual da Paraíba, Centro de Ciências Biológicas e Sociais Aplicadas, Departamento de Biologia, Laboratório de Ecologia, Rua Horácio Trajano de Oliveira, Cristo Redentor 58071-160, João Pessoa, Paraíba, Brasil. E-mail: clebsal@gmail.com

(4) Universidade Federal do Acre, Centro de Ciências Biológicas e da Natureza, Programa de PósGraduação em Ecologia e Manejo de Recursos Naturais, Rodovia BR-364 - km 04, Distrito Industrial 69920-223, Rio Branco, Acre, Brasil.

Amaranto I., Salimon C.I. \& Zeppelini D. (2018) Relação entre massa corporal e taxa respiratória em Arthropoda de Floresta Atlântica (Paraíba, Brasil). Pesquisa e Ensino em Ciências Exatas e da Natureza, 2(2): 119-129. http://dx.doi.org/10.29215/pecen.v2i2.1066

Editor acadêmico: Silvio F. B. Lima. Recebido: 15 Maio 2018. Aceito: 14 Setembro 2018. Publicado: 28 Dezembro 2018.

Resumo: Estudos de taxa respiratória em artrópodes remetem a dados eco fisiológicos importantes, uma vez que estes animais realizam importantes funções ecossistêmicas e por serem a maior diversidade e abundância de animais presentes na Terra. Esta pesquisa mede e descreve taxas respiratórias, através da emissão de $\mathrm{CO}_{2}$ e as relaciona com a massa corporal de artrópodes. Este estudo foi realizado em fragmentos remanescentes de Floresta Atlântica litorânea da Paraíba, Brasil. A pesquisa abrange todos os subfilos de artrópodes (Crustacea, Chelicerata, Myriapoda e Hexapoda) representados por 705 espécimes coletados ativamente entre outubro e dezembro de 2016 e experimentados através de respirometria fechada pelo Analisador de Gás por Infravermelho (IRGA). Como resultado foi visto que dentre os artrópodes avaliados os Hexapoda e Chelicerata apresentaram maiores taxas de emissão de dióxido de carbono enquanto que os Crustacea e Myriapoda as menores. Sobre a relação com a massa corporal, os Hexapoda e Crustacea apresentaram maior respiração por massa específica de artrópode, e em geral, todos os Arthropoda seguiram o padrão descrito pela fisiologia animal, onde a massa corporal e a taxa respiratória animal encontram-se correlacionadas inversamente.

Palavras chave: Artrópode terrestre, dióxido de carbono, respirometria animal, Analisador de Gás por Infravermelho.

Relation between body mass and respiratory rate in Arthropod of Atlantic Forest (Paraíba, Brazil)

Abstract: Respiratory rate studies in arthropods refer to important physiological data, since these animals perform important ecosystem functions and because they are the greatest diversity and abundance of animals present on Earth. This study measures and describes respiratory rates through the emission of $\mathrm{CO}_{2}$, and relates them to the body mass of arthropods. The study was carried out in remnant fragments of coastal Atlantic Forest of Paraíba, Brazil. The research encompasses all living subphyla of arthropods (Crustacea, Chelicerata, Myriapoda and Hexapoda) represented by 705 specimens actively collected and tested through respirometry and measured by Infrared Gas Analyzer (IRGA). As a result, we observed that among the studied arthropods, Hexapoda and Chelicerata showed higher rates of carbon dioxide emission, while Crustacea and Myriapoda had the lowest rates. Regarding its relationship with body mass, Hexapoda and Crustacea showed higher respiration by specific mass and, in general, all Arthropoda corroborated the 
pattern described by animal physiology, where body mass and animal respiratory rate are inversely correlated.

Key words: Terrestrial arthropod, carbon dioxide, animal respirometry, Infrared Gas Analyzer.

\section{Introdução}

A taxa metabólica é determinada a partir do consumo de moléculas de gás oxigênio ou da produção de moléculas de gás carbônico, portanto trata-se de uma forma quantitativa, precisa e prática de avaliar o desempenho fisiológico de um organismo (Harvey \& Brown 1951; Sustr \& Simek 1994; Brown et al. 2004). Para Brown e colaboradores (2004) a taxa metabólica é a taxa biológica mais relevante, e dentre os organismos heterótrofos, como os animais, incluindo os artrópodes, esta taxa remete-se à taxa respiratória. Define-se taxa respiratória como uma proporção de troca gasosa possível de ser quantificada na qual um animal necessita para atender às demandas impostas pelo ambiente (Peters 1983).

O estudo da taxa metabólica refere-se, também, a Biologia Geral onde, por exemplo, animais que apresentam taxa respiratória mais baixa tendem a possuir maior porte, apresentando uma potência respiratória (por massa específica) menor. Já animais que apresentam taxa respiratória mais alta, geralmente caracterizam-se como animais de porte menor, com potência respiratória por massa específica maior (Hsia et al. 2013). Um método para medir trocar gasosa realizada por animais é conhecido como respirometria, no qual utiliza-se a diferença de concentrações entre os gases respiratórios $\left(\mathrm{O}_{2}\right.$ e $\left.\mathrm{CO}_{2}\right)$ para calcular sua taxa de respiração (Hill et al. 2012). A taxa respiratória, por ser uma resposta imediata a um determinado fator exposto ao animal, podendo ser empregada na previsão de alterações fisiológicas em espécies animais (Macieira \& Proni 2005).

Representando a maior diversidade e abundância no planeta, os artrópodes oferecem amplos serviços ecossistêmicos abrangendo vários nichos em seus habitats, portanto são fundamentais nos estudos de avaliação da situação ambiental (Gaston 1991; Brusca \& Brusca 2007). Artrópodes traqueados apresentam a capacidade de controle de fluxo gasoso, originando padrões diferentes de trocas gasosas, bastante estudados em gafanhoto-do-deserto (Schistocerca gregaria), alguns miriápodes (Cormocephalus morsitans e Comantenna brevicornis) e até em alguns quelicerados, como os carrapatos (Cheliceriformes: Acari) e solífugos (Cheliceriformes: Solpugida), os quais apresentam um ciclo descontínuo de troca gasosa (DCG - do inglês, discontinuous gas-exchange cycles), onde é visto três fases da respiração traqueal: a fase fechada, onde há troca insignificante ou nenhuma entre o ambiente e o interior do corpo; a fase de vibração, onde os espiráculos abrem e fecham rapidamente, mantendo pequeno fluxo de $\mathrm{O}_{2}, \mathrm{CO}_{2}$ e vapor de água, difundindo-se no sistema respiratório animal; e a fase aberta, onde ocorre captação de $\mathrm{O}_{2}$ do ambiente e o escape de $\mathrm{CO}_{2}$ e vapor de água do metabolismo animal (Lighton et al. 1993; Lighton \& Fielden 1995, 1996; Lighton 1998; Chappell \& Rogowitz 2000; Lehmann 2001; Shelton \& Appel 2001; Klok et al. 2002; Huang et al. 2014). A diferença de concentração dos gases respiratórios (principalmente o $\mathrm{O}_{2}$ ) movimenta a abertura e fechamento espiracular destes animais, onde a baixa pressão intratraqueal de $\mathrm{O}_{2}$ resulta na abertura dos espiráculos e a alta pressão intratraqueal de $\mathrm{O}_{2}$ em seu fechamento (Lighton et al. 2004; Förster \& Hetz 2010; Matthews et al. 2012).

Alterações fisiológicas interferem consideravelmente no metabolismo animal, como alterações na massa corporal, uma vez que animais pequenos respiram em taxas metabólicas mais elevadas por unidade de massa corporal, do que animais grandes, como observado em estudos com relação biomassa e taxa respiratória em gafanhoto (Locusta migratoria), ou seja, os dados apresentaram uma relação alométrica negativa com a massa corporal do inseto (Bertalanffy 1957; Greenlee et al. 2007; Randall et al. 2011; Forster et al. 2012).

Há fatores ambientais que influenciam diretamente o fluxo respiratório de animais, como é o exemplo da temperatura, na qual o seu aumento eleva a taxa de respiração em abelhas (Trigona spinipes) e em colêmbolos (Tetrodontophora bielanensis) (Zinkler 1966; Sustr 1996; 
Brown et al. 2004; Macieira \& Proni 2005). Outros fatores, como o estado alimentado de animais ou o período de crescimento sazonal afetam diretamente a atividade respiratória no qual animais em jejum ou em fase de repouso (geada ou seca) tendem a apresentar taxas de respiração mais baixas (Cloudsley-Thompson 1975; Zettel 1982; Woude \& Joosse 1988). Em espécies que realizam mudas periódicas, os intervalos entre as mudas apresentam elevada taxa metabólica, uma vez que não ocorre o aumento no tamanho das estruturas esclerotizadas de seu sistema de troca gasosa (Greenlee \& Harrison 2004). Além do mais, determinados fatores intrínsecos da espécie, como sua ontogenia, afetarem diretamente o padrão das taxas respiratórias. Neste sentido, colêmbolos juvenis, por exemplo, tendem a apresentar maiores taxas de consumo de $\mathrm{O}_{2}$ do que adultos da mesma espécie (Hopkin 1997).

Desta forma, como as taxas respiratórias apresentam padrões diversos, devido tanto a variações ambientais como intrínsecas dos organismos, estudos voltados para respiração animal são capazes de produzir informações acerca de sua fisiologia e ecofisiologia, podendo fornecer uma medida de custo energético entre animal e ecossistema (Peters 1983).

Estudos descritivos da respiração por massa específica de artrópode permite o desenvolvimento de uma condição mais ampla à ciência aplicada a este filo de grande importância ecológica e econômica (Klein et al. 2007; Richardson 2008). Esta pesquisa pretende determinar a taxa respiratória, através da emissão de $\mathrm{CO}_{2}$ e correlacioná-la com a massa corporal de representantes dos maiores grupos de artrópodes, uma vez que apresentam modo de vida diferentes, mecanismos respiratórios diferentes. Estudos de ciência básica envolvendo tais metazoários e a abordagem aqui apresentada são ainda escassos, consequentemente, havendo poucos dados na literatura científica.

\section{Material e Métodos}

\section{Área de coleta}

Esta pesquisa foi realizada em áreas de Mata Atlântica no Estado da Paraíba (Figura 1). O primeiro ponto de coleta ocorreu em pequenas áreas verdes urbanas contidas no Campus João Pessoa da Universidade Estadual da Paraíba (UEPB), situada na mesma cidade, no qual apresenta aproximadamente 2 hectares de área $(-7.159077 \mathrm{~S},-34.872860 \mathrm{~W})$. O segundo ponto ocorreu no fragmento Mata do Buraquinho que contém cerca de 515 hectares de área (SEMAN 2012), considerado o maior fragmento de Mata Atlântica da cidade de João Pessoa (-7.145764 S, $34.855885 \mathrm{~W})$. O terceiro ponto de coleta ocorreu em um fragmento da Área de Proteção Ambiental (APA) de Tambaba (-7.326503 S, -34.801813 W), situado na cidade de Conde - Paraíba, com área de 11.320 hectares (SUDEMA 2012), Decreto Estadual PB nº 22.882.

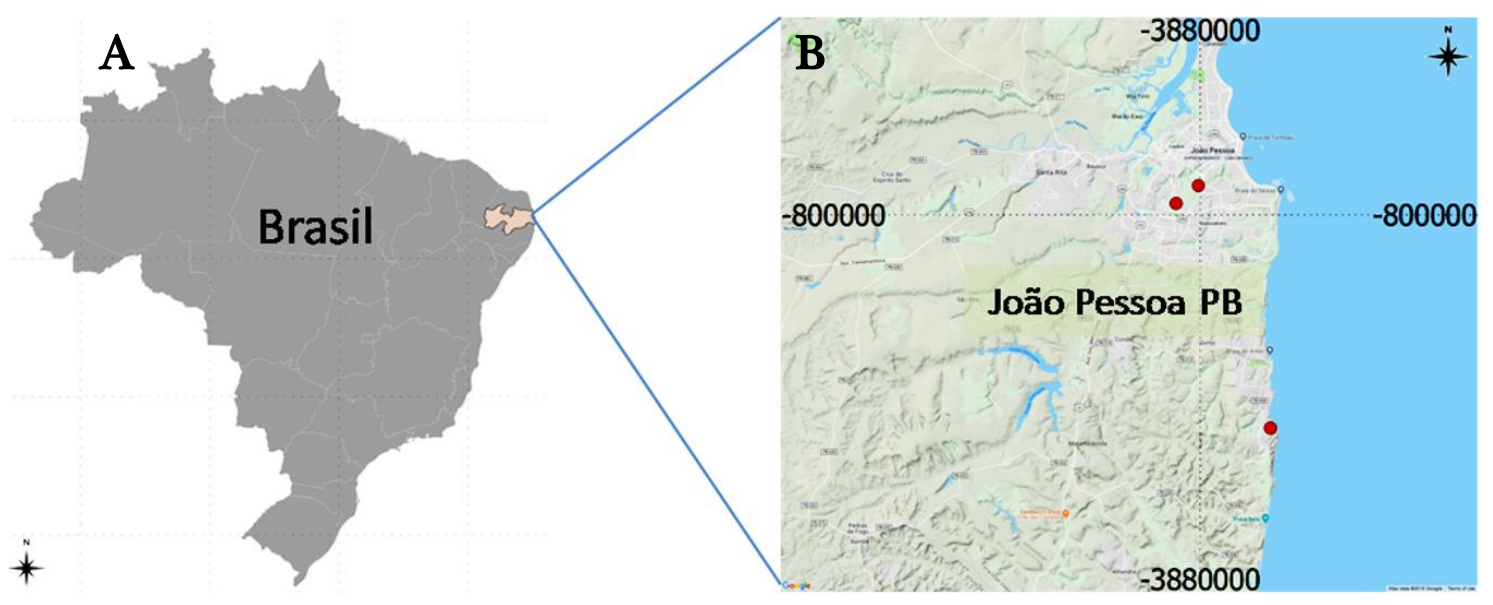

Figura 1. (A) Mapa do Brasil destacando o Estado da Paraíba; (B) Mapa mostrando trecho do Estado da Paraíba e os locais de coleta de artrópodes (pontos vermelhos) em região de Floresta Atlântica, no município de João Pessoa, Paraíba (coordenadas em Universal Transversa de Mercator - UTM; e a camada de elevação do terreno do Google@2018 - https://earth.google.com). 


\section{Coleta e Aclimatação}

Setecentos e cinco (705) espécimes representantes dos quatro subfilos de Arthropoda foram coletados, de forma aleatória (Tabela 1), durante o último trimestre de 2016. A temperatura na área de estudo oscilou entre 22.1 e $32^{\circ} \mathrm{C}$, durante o período de coleta, segundo os dados encontrados na plataforma do Instituto Nacional de Pesquisas Espaciais - INPE, referente ao mesmo período.

Tabela 1. Amostragem dos artrópodes terrestres de Mata Atlântica utilizados no estudo de respirometria. Ind. = indivíduos.

\begin{tabular}{lllccc}
\hline \multicolumn{1}{c}{ Táxon de Arthropoda } & Localização de coleta & Coordenada de coleta & Precipitação & $\begin{array}{c}\text { Massa } \\
\text { corporal }\end{array}$ & Ind. \\
\hline Isopoda & Áreas verdes da UEPB-V & $7.158834 \mathrm{~S}, 34.873401 \mathrm{~W}$ & $1327.65 \mathrm{~mm}$ & $0.320 \mathrm{~g}$ & 10 \\
Coleoptera & APA de Tambaba & $7.193302 \mathrm{~S}, 34.48539 \mathrm{~W}$ & $783.3 \mathrm{~mm}$ & $0.160 \mathrm{~g}$ & 01 \\
Isoptera & Mata do Buraquinho & $7.142905 \mathrm{~S}, 34.845957 \mathrm{~W}$ & $1327.65 \mathrm{~mm}$ & $1.200 \mathrm{~g}$ & 677 \\
Orthoptera & Áreas verdes da UEPB-V & $7.158834 \mathrm{~S}, 34.873401 \mathrm{~W}$ & $1327.65 \mathrm{~mm}$ & $0.940 \mathrm{~g}$ & 01 \\
Hymenoptera (Formicidae) & Mata do Buraquinho & $7.142905 \mathrm{~S}, 34.845957 \mathrm{~W}$ & $1327.65 \mathrm{~mm}$ & $0.560 \mathrm{~g}$ & 02 \\
Hymenoptera (Vespidae) & APA de Tambaba & $7.193988 \mathrm{~S}, 34.483650 \mathrm{~W}$ & $783.3 \mathrm{~mm}$ & $0.720 \mathrm{~g}$ & 01 \\
Diptera & APA de Tambaba & $7.193988 \mathrm{~S}, 34.483650 \mathrm{~W}$ & $783.3 \mathrm{~mm}$ & $0.130 \mathrm{~g}$ & 01 \\
Aracnida (Ixodidae) & Mata do Buraquinho & $7.142905 \mathrm{~S}, 34.845957 \mathrm{~W}$ & $1327.65 \mathrm{~mm}$ & $3.230 \mathrm{~g}$ & 01 \\
Aracnida (Scorpiones) & Áreas verdes da UEPB-V & $7.158834 \mathrm{~S}, 34.873401 \mathrm{~W}$ & $1327.65 \mathrm{~mm}$ & $0.310 \mathrm{~g}$ & 01 \\
Aracnida (Araneae) & APA de Tambaba & $7.193302 \mathrm{~S}, 34.48539 \mathrm{~W}$ & $783.3 \mathrm{~mm}$ & $0.288 \mathrm{~g}$ & 01 \\
Diplopoda & Áreas verdes da UEPB-V & $7.158834 \mathrm{~S}, 34.873401 \mathrm{~W}$ & $1327.65 \mathrm{~mm}$ & $1.940 \mathrm{~g}$ & 07 \\
Chilopoda & APA de Tambaba & $7.193302 \mathrm{~S}, 34.48539 \mathrm{~W}$ & $783.3 \mathrm{~mm}$ & $0.220 \mathrm{~g}$ & 02 \\
\hline
\end{tabular}

Para os Miryapoda abrangemos o grupo dos milípedes (Diplopoda) e o das centopeias (Chilopoda). No subfilo dos Chelicerata foram realizados experimentos com os aracnídeos: aranha (Nephilidae), escorpião (Buthidae) e carrapato (Ixodidae). Dentre os Hexapoda foram coletadas formigas e vespa (Hymenoptera), mosca (Diptera), grilo (Orthoptera), besouro (Coleoptera) e cupins (Isoptera). Dentre os crustáceos terrestres, escolhemos os tatuzinhos-dejardim (Isopoda).

Posteriormente a coleta, os espécimes foram acondicionados em recipientes aerados e encaminhados ao Laboratório de Sistemática de Collembola e Conservação (LSCC) da UEPB-V. Os espécimes foram aclimatados por cerca de 60 minutos à $29^{\circ} \mathrm{C}$ e umidade relativa de $85 \% \mathrm{em}$ uma sala anexa ao LSCC que possui temperatura e umidade controladas, para reduzir quaisquer efeitos de estresse animal.

\section{Respirometria e Medição de Massa Corporal}

Os espécimes foram colocados em uma câmara de respiração totalmente vedada, com 4 cm de diâmetro, $5.55 \mathrm{~cm}$ de altura e $0.09 \mathrm{~L}$ de volume. Todos os representantes de cada táxon foram analisados juntos, para a realização da respirometria do grupo, totalizando 12 experimentos independentes de respirometria animal. Os ensaios de respirometria foram realizados com concentrações normais de gases atmosféricos.

A câmara foi interligada diretamente ao Analisador de Gás por Infravermelho (IRGA) LI-840 Gas Analyzer®. O experimento resultou em um sistema fechado entre o analisador de gás e a câmara de respiração.

O estudo aqui realizado com o IRGA aplicado a respirometria animal avaliando a emissão de dióxido de carbono para cálculo de taxa respiratória está baseado em Lighton (1990), Chown (2001) e Withers (2001).

Após medições com durações de até 10 minutos, em uma única sessão de respirometria por grupo, os espécimes foram retirados da câmara e, através de uma balança de precisão foi medida as massas corporais dos grupos (Schimpf et al. 2009). 


\section{Análise estatística}

$\mathrm{O}$ analisador de gás mediu a concentração de dióxido de carbono $\left(\mathrm{CO}_{2}\right)$ contido na câmara de respiração, durante o metabolismo respiratório do animal, onde os valores foram coletados em arquivo " $t x t$ " a cada segundo e plotados em planilhas eletrônicas. Através das planilhas de dados de respirometria foram calculados os valores da taxa respiratória para os 12 táxons de artrópodes experimentados, segundo a Equação 1 (Davidson \& Trumbore 1995),

$$
\text { FluxoCO } 2\left(m g \mathrm{CO}_{2} l^{-1} h^{-1}\right)=\frac{p \mathrm{CO}_{2}}{T * m_{\text {ol }}} \times \frac{\mathrm{Var}_{\text {ar }}}{\text { tempo }} \times \frac{d\left[\mathrm{CO}_{2}\right]}{d t}
$$

onde: $\mathrm{pCO}_{2}$ - concentração parcial de $\mathrm{CO}_{2} ; \mathrm{T}$ - temperatura em Kelvin; $\mathrm{mol}_{\mathrm{ar}}$ - moles de ar; $\mathrm{V}_{\mathrm{ar}}$ - volume de ar; $\mathrm{d}\left[\mathrm{CO}_{2}\right]$ - variação na concentração de $\mathrm{CO}_{2} ; \mathrm{dt}$ - intervalo de tempo.

Regressões lineares foram feitas para cada táxon, baseando-se no período de acúmulo de $\mathrm{CO}_{2}$. As regressões foram testadas estatisticamente através de ANOVA do coeficiente angular da reta, com o pacote Analysis Toolpak do Microsoft Excel @ 2016.

A relação entre massa corporal e taxa respiratória foi testada através de ajuste de curvas, no software Bioestat ${ }^{\circledR} 5.3$, para determinar a forma e a significância da relação, utilizando-se os dados dos doze ensaios de respirometria realizados no Microsoft Excel ${ }^{\circledR} 2016$.

\section{Resultados e Discussão}

\section{Taxa respiratória por emissão de $\mathrm{CO}_{2}$}

A Figura 2 apresenta o acúmulo de $\mathrm{CO}_{2}$ no decorrer do tempo de experimento de respirometria para cada táxon de Arthropoda. As curvas de regressões a seguir apresentam nível de significância $\mathrm{p}<0.05$ e seus valores de determinação $\left(\mathrm{R}^{2}\right)$ explícitos em cada gráfico (Figura 2).

Na Tabela 2 é visto o resumo dos resultados obtidos para os experimentos de respirometria realizados nos 12 táxons de Arthropoda.

Tabela 2. Taxa de respiração através de fluxo de $\mathrm{CO}_{2}$ em artrópodes terrestres de Mata Atlântica, onde a taxa respiratória em $m g: C O_{2} m^{3} \cdot h^{-1}$ (massa corporal em g).

\begin{tabular}{lccc}
\hline \multicolumn{1}{c}{ Táxon de Arthropoda } & Taxa respiratória & Massa Corporal & $\begin{array}{c}\text { Razão fluxo de } \mathbf{C O}_{2} \\
\text { e massa corporal }\end{array}$ \\
\hline CRUSTACEA & 1527.3 & 0.32 & 4772.81 \\
Isopoda & & & \\
HEXAPODA & 1905.7 & 0.16 & 11910.63 \\
Coleoptera & 2412.8 & 1.20 & 2010.67 \\
Isoptera & 1299.2 & 0.94 & 1382.13 \\
Orthoptera & 1141.7 & 0.56 & 2038.75 \\
Hymenoptera (Formicidae) & 5063.5 & 0.72 & 7032.64 \\
Hymenoptera (Vespidae) & 13903.0 & 0.13 & 106946.15 \\
Diptera & & & 2090.19 \\
CHELICERIFORMES & 6751.3 & 3.23 & 3878.39 \\
Aracnida (Ixodidae) & 1202.3 & 0.31 & 2285.07 \\
Aracnida (Buthidae) & 658.1 & 0.288 & \\
Aracnida (Nephilidae) & & & 281.39 \\
MYRIAPODA & 545.9 & 1.94 & 3173.18 \\
Diplopoda & 698.1 & 0.22 & \\
Chilopoda & & & \\
\hline
\end{tabular}




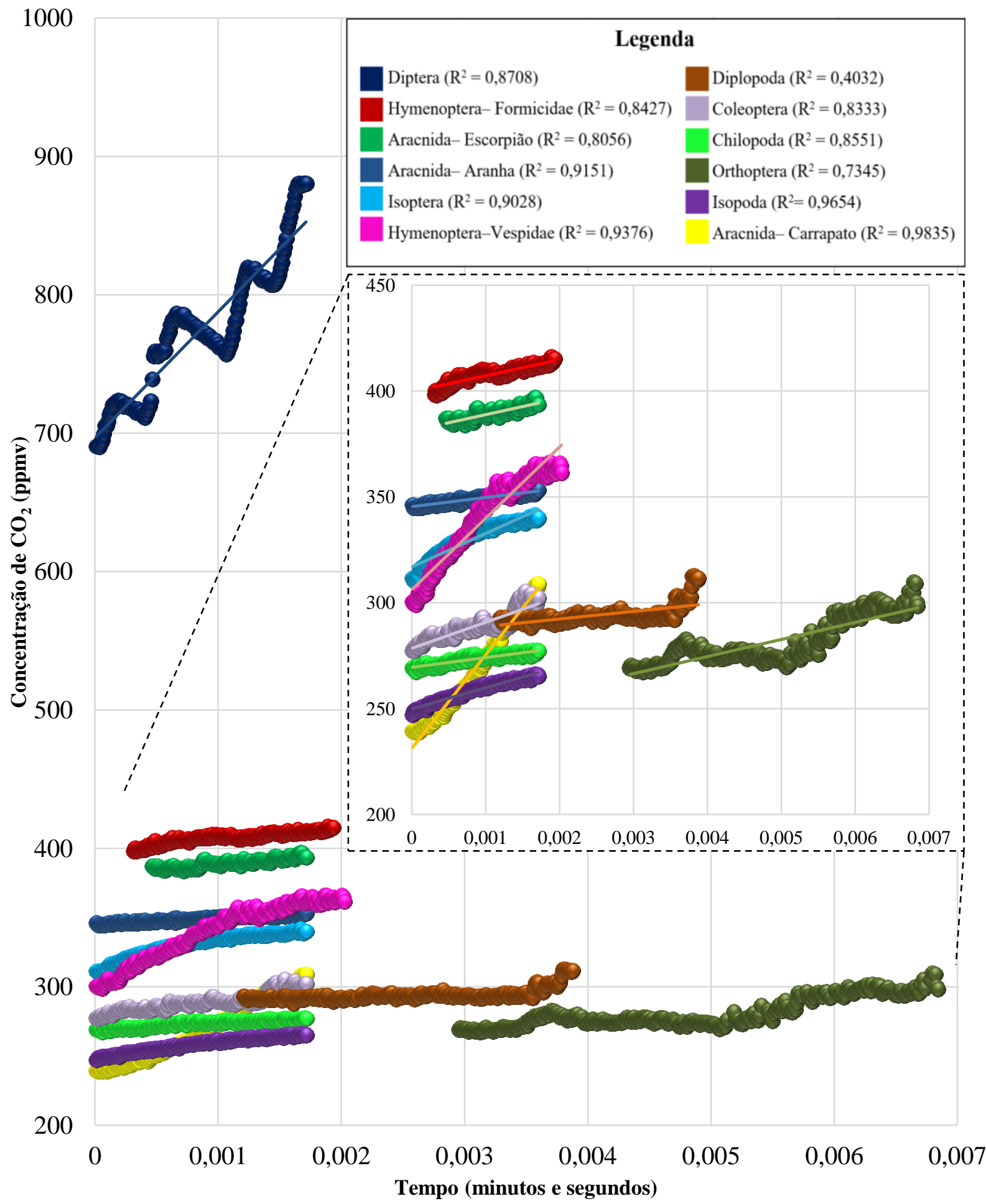

Figura 2. Variação da concentração de $\mathrm{CO}_{2}$ ao longo de tempo para diferentes Arthropoda de Mata Atlântica com os respectivos $\mathrm{r}^{2}$ das regressões entre concentração e tempo.

Os artrópodes analisados obtiveram taxas distintas de respiração, onde a menor taxa respiratória foi de Diplopoda (545.9 $\left.\mathrm{mg} \cdot \mathrm{CO}_{2} \cdot \mathrm{m}^{3} . h\right)$, representante do subfilo Miryapoda; a maior foi de Diptera (13903.0 $\mathrm{mg} \cdot \mathrm{CO}_{2} \mathrm{~m}^{3} . h$ ), representante do subfilo Hexapoda; enquanto que a média de respiração por emissão de $\mathrm{CO}_{2}$ dos artrópodes terrestres ficou em torno de 3092.41 $m g . C \mathrm{O}_{2} \cdot m^{3} \cdot h$. A Figura 3 apresenta a disposição das taxas respiratórias por emissão de $\mathrm{CO}_{2}$. 


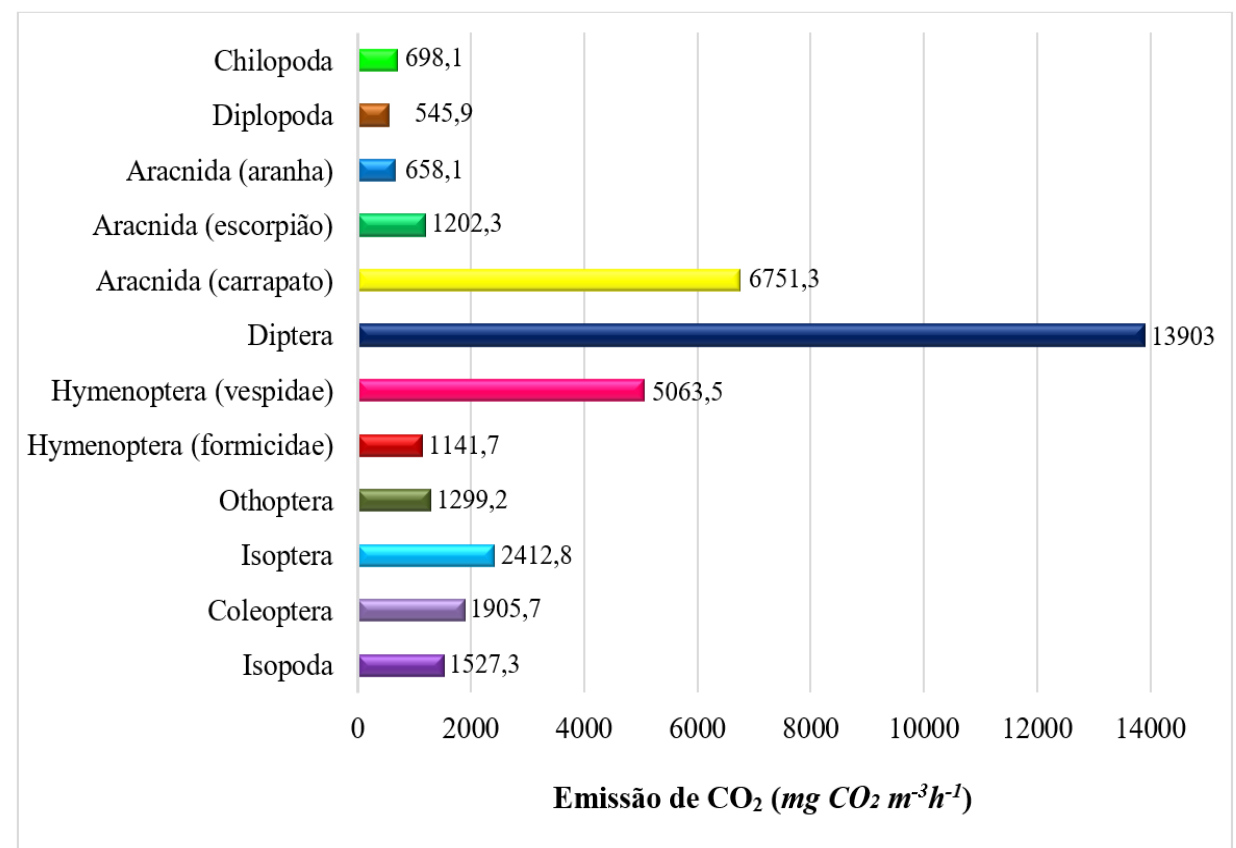

Figura 3. Taxas respiratórias através de emissão de $\mathrm{CO}_{2}$ em Arthropoda terrestre de Mata Atlântica.

\section{Relação taxa respiratória por emissão de $\mathrm{CO}_{2}$ e Massa Corporal}

Como visto na Tabela 2, todos os espécimes apresentaram diferentes massas corporais, onde o táxon de maior massa foi Cheliceriforme - Aracnida (3.230 gramas) representado por um carrapato fêmea; o táxon de menor massa corporal foi Hexapoda - Diptera (0.130 gramas); e a massa corporal média foi de 0.835 gramas.

$\mathrm{Na}$ Figura 4 observamos a normalização da emissão de $\mathrm{CO}_{2}$ por massa corporal específica de artrópodes, ajuste necessário para correlacionar a taxa de respiração com a massa corporal.

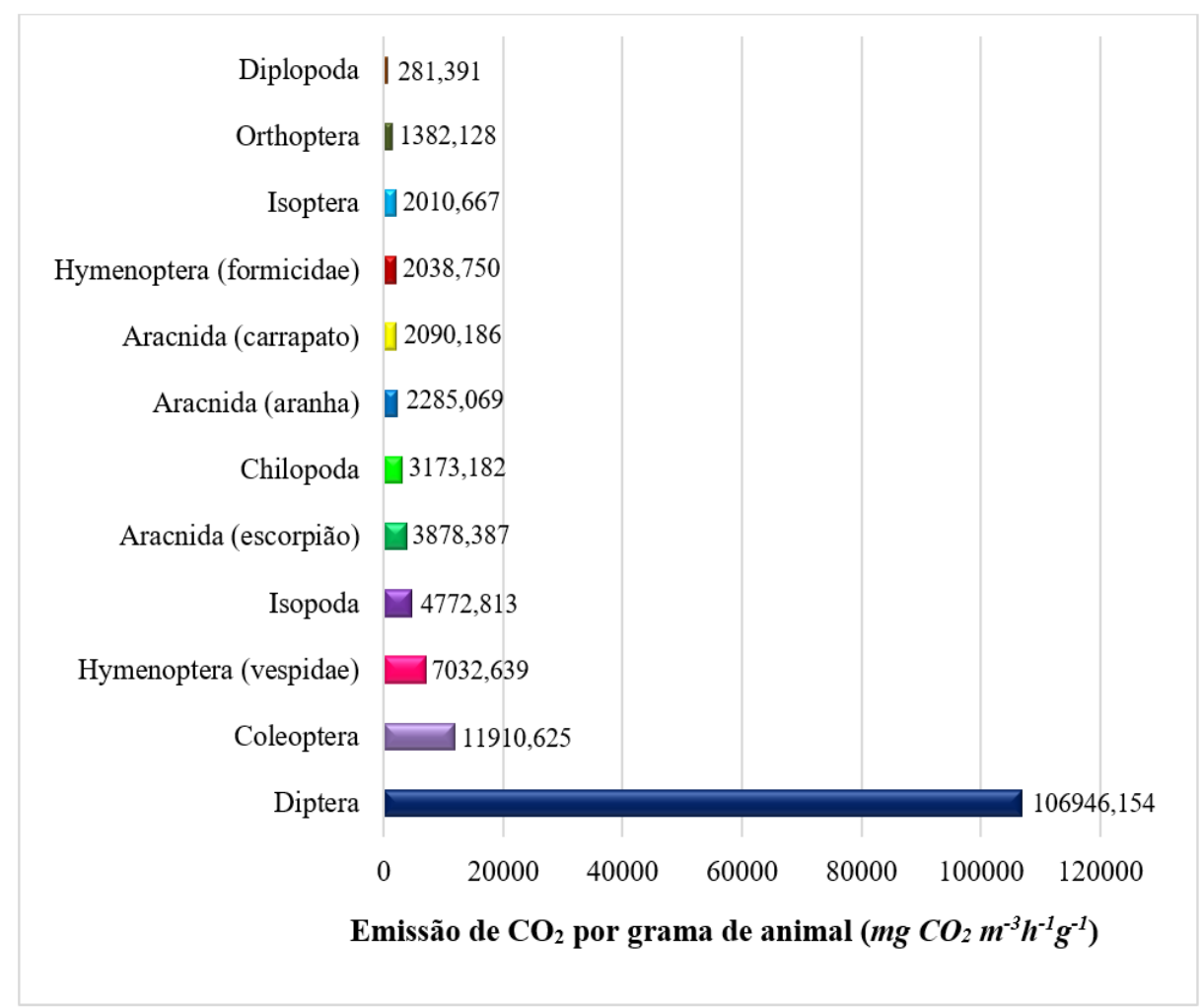

Figura 4. Normalização da taxa respiratória (razão: Emissão de $\mathrm{CO}_{2}$ /grama de animal). 
A regressão geométrica da taxa de emissão de $\mathrm{CO}_{2}$ normalizada por unidade de massa corporal de Arthropoda com $r^{2}=0.5051$ e $p=0.0095$ é vista na Figura 5, na qual foi encontrada uma relação inversa entre a respiração por emissão de $\mathrm{CO}_{2}$ e a massa corporal dos artrópodes, onde o Aracnida - Carrapato por apresentar maior massa corporal dentre os outros táxons correspondeu a uma taxa respiratória por massa específica bastante reduzida, porém maior do que o Myriapoda - Diplopoda, no qual apresentou a segunda maior massa corporal analisada, refletindo assim no mais reduzido metabolismo respiratório. Já o Hexapoda - Diptera por apresentar a menor massa corporal, segue o padrão da fisiologia animal apresentando, assim, a maior taxa respiratória por grama de animal.

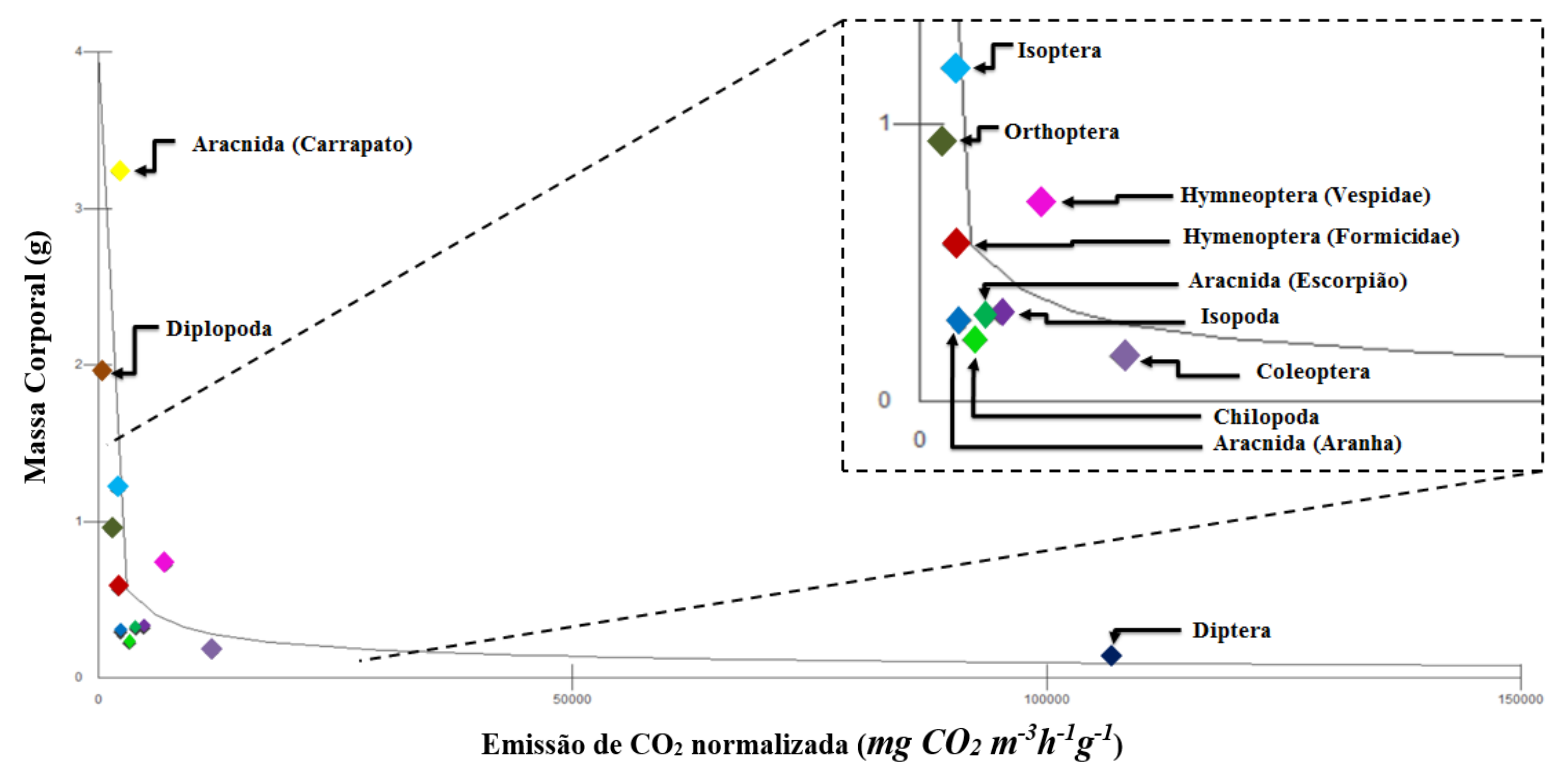

Figura 5. Taxa de respiração por unidade de massa corporal de Arthropoda terrestre de Mata Atlântica, com ampliação detalhada para a curva.

As taxas respiratórias por emissão de $\mathrm{CO}_{2}$ e as massas corporais de artrópodes apresentaram-se inversamente relacionadas (Makarieva et al. 2008; Randall et al. 2011), uma vez que os táxons de menores massas apresentaram maiores taxas metabólicas de emissão de $\mathrm{CO}_{2} \mathrm{e}$ o inverso foi visto nos exemplares dos táxons com maiores massas corporais. Uma vez que o tamanho do corpo dos artrópodes aumenta, a sua taxa metabólica absoluta segue o mesmo padrão, já que quanto maior o animal, maior também a sua demanda energética total (SchmidtNielsen 1984), porém a sua taxa metabólica específica de massa diminui.

A massa corporal é um dos principais atributos que se relaciona com a aptidão individual provocando adaptações comportamentais e fisiológicas, como alterações na respiração animal (Hildrew et al. 2007). Assim, uma forma de compensar o tamanho aumentado do corpo de certos artrópodes é alterando os mecanismos ventilatórios, como o volume de ar respiratório captado pelo animal ou a sua frequência de captação (Greenlee et al. 2007).

O estudo forneceu a primeira descrição de taxas respiratórias de grupos de artrópodes de Floresta Atlântica e expôs a existência de uma relação negativa entre a respiração e massa corporal, no qual serve como base para estudos específicos de metabolismo de artrópodes, estudos de ecologia de fauna de solo ou até explanações acerca da emissão de $\mathrm{CO}_{2}$ por artrópodes terrestres, além de estudos de Ecofisiologia animal. Evidencia ainda que impactos nestas comunidades de meso a macrofauna terrestres são importantes de serem avaliados, pois a fauna de solo regula e promove as funções ambientais vitais, uma vez que muitas espécies de artrópodes são conhecidas como engenheiros de ecossistema e espécies-chave para a estrutura do ecossistema, no qual podem desequilibrar os fluxos de recursos no ambiente por serem sensíveis às alterações ambientais (Wallwork 1970; Brussaard 1997; Wolters et al. 2000; Smith et al. 2009). 


\section{Agradecimentos}

Aos avaliadores anônimos pela revisão crítica do manuscrito.

\section{Referências}

Bertalanffy L. VON. (1957) Quantitative laws in metabolism and growth. The Quarterly Review of Biology, 32(3): 217-231.

Brown J.H., Gillooly J.F., Allen A.P., Savage V.M. \& West G.B. (2004) Toward a metabolic theory of Ecology. Ecology, 85(7): 1771-1789. DOI: 10.1890/03-9000

Brusca G.J. \& Brusca R.C. (2007) Invertebrados. $2^{\circ}$ edição. Rio de Janeiro: Guanabara Koogan. 968 p.

Brussaard L. (1997) Biodiversity and Ecosystem Functioning in Soil. Ambio, 26(8): 563-570.

Chappell M.A. \& Rogowitz G.L. (2000) Mass, temperature and metabolic effects on discontinuous gas exchange cycles in eucalyptus-Boring beetles (Coleoptera: Cerambycidae). The Journal of Experimental Biology, 203: 3809-3820.

Chown S.L. (2001) Physiological variation in insects: hierarchical levels and implications. Journal of Insect Physiology, 47(7): 649-660.

Cloudsley-Thompson J.L. (1975) Adaptations of Arthropoda to Arid Environments. Annual Review of Entomology, 20: 261-283. DOI: 10.1146/annurev.en.20.010175.001401

Davidson E.A. \& Trumbore S.E. (1995) Gas diffusivity and production of $\mathrm{CO}_{2}$ in deep soils of the eastern Amazon. Tellus, 47: 550-565. DOI: 10.3402/tellusb.v47i5.16071

Forster J., Hirst A.G. \& Atkinson D. (2012) Warming-induced reductions in body size are greater in aquatic than terrestrial species. Proceedings of the National Academy of Sciences of the United States of America, 109(47): 19310-19314. DOI: 10.1073/pnas.1210460109

Förster T.D. \& Hetz S.K. (2010) Spiracle activity in moth pupae-The role of oxygen and carbon dioxide revisited. Journal of Insect Physiology, 56(5): 492-501. DOI: 10.1016/j.jinsphys.2009.0 6.003

Gaston K.J. (1991) The Magnitude of Global Insect Species Richness. Conservation Biology, 5(3): 283-296.

Greenlee K.J. \& Harrison J.F. (2004) Development of respiratory function in the American locust Schistocerca americana. I. Across-instar effects. Journal of Experimental Biology, 207(3): 497508. DOI: $10.1242 /$ jeb.00767

Greenlee K.J., Nebeker C. \& Harrison J.F. (2007) Body size-independent safety margins for gas exchange across grasshopper species. The Journal of Experimental Biology, 210: 1288-1296. DOI: $10.1242 /$ jeb.001982

Harvey G.T. \& Brown A.W.A. (1951) The effect of insecticides on the rate of oxygen consumption in Blattella. Canadian Journal of Zoology, 29(1): 42-53. DOI: 10.1139/z51-004

Hildrew A.G., Raffaelli D.G. \& Edmonds-Brown R. (2007) Body Size: The Structure and Function of Aquatic Ecosystems. Ecological Reviews. Cambridge: Cambridge University Press. DOI: 10.1017/CBO9780511611223

Hill R.W., Wyse G.A. \& Anderson M. (2012) Fisiologia Animal. $2^{\circ}$ edição. Porto Alegre: Porto Alegre. 920 p.

Hopkin S.P. (1997) Biology of the Springtails (Insecta: Collembola). Oxford: Oxford University Press. 340 p.

Hsia C.C., Schmitz A., Lambertz M., Perry S.F. \& Maina J.N. (2013) Evolution of Air Breathing: Oxygen Homeostasis and the Transitions from Water to Land and Sky. Comprehensive Physiology, 3(2): 849-915. DOI: 10.1002/cphy.c120003

Huang S.P., Sender R. \& Gefen E. (2014) Oxygen diffusion limitation triggers ventilatory movements during spiracle closure when insects breathe discontinuously. Journal of Experimental Biology, 217: 2229-2231. DOI: 10.1242/jeb.102426

Klein A.-M., Vaissière B.E., Cane J.H., Steffan-Dewenter I., Cunningham S.A., Kremen C. \& Tscharntke T. (2007) Importance of pollinators in changing landscapes for world crops. 
Proceedings of the Royal Society B: Biological Sciences, 274(1608): 303-313. DOI:10.1098/rspb.2006.3721

Klok C.J., Mercer R.D. \& Chown S.L. (2002) Discontinuous gas-exchange in centipedes and its convergent evolution in tracheated arthropods. The Journal of Experimental Biology, 205(7): 1019-1029.

Lehmann F.-O. (2001) Matching Spiracle Opening to Metabolic Need During Flight in Drosophila. Science, 294(5548): 1926-1929. DOI: 10.1126/science.1064821

Lighton J.R.B. (1990) Slow discontinuous ventilation in the namib dune-sea ant Camponotus detritus (Hymenoptera, Formicidae). Journal of Experimental Biology, 151: 71-82.

Lighton J.R.B. (1998) Notes from Underground: Towards Ultimate Hypotheses of Cyclic, Discontinuous Gas-Exchange in Tracheate Arthropods. American Zoologist, 38(3): 483-491.

Lighton J.R.B. \& Fielden L.J. (1995) Mass Scaling of Standard Metabolism in Ticks: A Valid Case of Low Metabolic Rates in Sit-and-Wait Strategists. Physiological Zoology, 68(1): 43-62. DOI: $10.2307 / 30163917$

Lighton J.R.B. \& Fielden L.J. (1996) Gas exchange in wind spiders (Arachnida, Solphugidae): independent evolution of convergent control strategies in solphugids and insects. Journal of Insect Physiology, 42(4): 347-357. DOI: 10.1016/0022-1910(95)00112-3

Lighton J.R.B., Fielden L.J. \& Rechav Y. (1993) Discontinuous ventilation in a non-insect, the tick Amblyomma marmoreum (Acari, Ixodidae): characterization and metabolic modulation. Journal of Experimental Biology, 180: 229-245.

Lighton J.R.B., Schilman P.E. \& Holway D.A. (2004) The hyperoxic switch: assessing respiratory water loss rates in tracheate arthropods with continuous gas exchange. Journal of Experimental Biology, 207: 4463-4471. DOI: 10.1242/jeb.01284

Macieira O.J.D. \& Proni E.A. (2005) Influência da temperatura na taxa respiratória de abelhas forrageiras Trigona spinipes (Fabricius) (Hymenoptera, Apidae, Meliponinae) durante períodos de verão e inverno. Revista Brasileira de Zoologia, 22(4): 1159-1163. DOI: 10.1590/S0101-81752005000400050.

Makarieva A.M., Gorshkov V.G., Li B.-L., Chown S.L., Reich P.B. \& Gavrilov V.M. (2008) Mean mass-specific metabolic rates are strikingly similar across life's major domains: Evidence for life's metabolic optimum. Proceedings of the National Academy of Sciences of the United States of America, 105(44): 16994-16999. DOI: 10.1073/pnas.0802148105

Matthews P.G.D., Snelling E.P., Seymour R.S. \& White C.R. (2012) A test of the oxidative damage hypothesis for discontinuous gas exchange in the locust Locusta migratoria. Biology Letters, 8: 682-684. DOI: $10.1098 / \mathrm{rsbl} .2012 .0137$

Peters R.H. (1983) The ecological implications of body size. Cambridge: Cambridge University Press. $234 \mathrm{p}$.

Randall D., Burggren W. \& French K. (2011) Fisiologia Animal: Mecanismos e Adaptações. $4^{\circ}$ edição. Rio de Janeiro: Guanabara Koogan. 764 p.

Richardson A.J. (2008) In hot water: zooplankton and climate change. ICES Journal of Marine Science, 65: 279-295.

Schimpf N.G., Matthews P.G.D., Wilson R.S. \& White C.R. (2009) Cockroaches breathe discontinuously to reduce respiratory water loss. The Journal of Experimental Biology, 212: 2773-2780.

Schmidt-Nielsen K. (1984) Scaling: Whyis Animal SizesoImportant? New York: Cambridge University Press. $241 \mathrm{p}$.

SEMAN - Secretaria do Meio Ambiente (2012) Plano Municipal de Conservação e Recuperação da Mata Atlântica. João Pessoa: Diretoria de Estudos e Pesquisas Ambientais. Disponível em: http://www.joaopessoa.pb.gov.br/secretarias/semam/plano-municipal-mata-atlantica/ (Acessado: 02/03/2018).

Shelton T.G. \& Appel A.G. (2001) Cyclic $\mathrm{CO}_{2}$ release in Cryptotermes cavifrons Banks, Incisitermes tabogae (Snyder) and I. minor (Hagen) (Isoptera: Kalotermitidae). Comparative Biochemistry and Physiology - Part A Molecular \& Integrative Physiology, 129(2): 681-693. DOI: $10.1016 / \mathrm{S} 1095-6433(01) 00332-4$ 
Smith J.J., Hasiotis S.T., Kraus M.J. \& Woody D.T. (2009) Transient dwarfism of soil fauna during the Paleocene-Eocene Thermal Maximum. Proceedings of the National Academy of Sciences of the United States of America, 106(42): 17655-17660. DOI: 10.1073/pnas.0909674106

SUDEMA - Superintendência de Administração do Meio Ambiente (2012) Área de Proteção Ambiental (APA) de Tambaba. João Pessoa: SUDEMA. Disponível em: http://www.sudema.pb .gov.br./index.php?view=category\&catid=5\&option=com_joomgallery (Acessado: 15/05/2018).

Sustr V. (1996) Influence of temperature acclimation on respiration-temperature relationship in Tetrodontophora bielanensis (Collembola: Onychiuridae). European Journal of Entomology, 93(3): 435-442.

Sustr V. \& Simek M. (1994) Environmental factors influencing respiration of the stenothermic collembolan Tetrodontophora bielanensis (Entognatha, Collembola). Pedobiologia, 38: 63-71.

Wallwork J.A. (1970) Ecology of Soil Animals. London, New York: McGraw-Hill. 283 p.

Withers P.C. (2001) Design, calibration and calculation for flow-through respirometry systems. Australian Journal of Zoology, 49: 445-461.

Wolters V., Silver W.L., Bignell D.E., Coleman D.C., Lavelle P., Van Der Putten W.H., De Ruiter P., Rusek J., Wall D.H., Wardle D.A., Brussaard L., Dangerfield J.M., Brown V.K., Giller K.E., Hooper D.U., Sala O., Tiedje J. \& Van Veen J.A. (2000) Effects of global changes on above- and belowground biodiversity in terrestrial Ecosystems: Implications for ecosystem functioning. BioScience, 50(12): 1089-1098.

Woude H.A. Van Der \& Joosse E.N.G. (1988) The seasonality of respiration in two temperate Collembola as related to starvation, temperature and photoperiod. Comparative Biochemistry and Physiology Part A Physiology, 91(1): 147-151. DOI: 10.1016/0300-9629(88)91607-6

Zettel J. (1982) Dietary influence on the biology and metabolism of Isotoma viridis (Collembola). Journal of Insect Physiology, 28(5): 453-458. DOI: 10.1016/0022-1910(82)900749

Zinkler D. (1966) Vergleichende Untersuchungen zur Atmungsphysiologie von Collembolen (Apterygota) und anderen Bodenkleinarthropoden. Zeitschrift für vergleichende Physiologie, 52(2): 99-144. 